\title{
Ethical Issues in Psychological Research in the Wake of COVID-19
}

\author{
Riddhi Laijwala ${ }^{1}$, Parena Sahukar ${ }^{2}$ \\ ${ }^{1,2}$ Research Assistant, Desousa Foundation, Mumbai \\ Corresponding Author: Riddhi Laijawala \\ E-mail: riddhi.laijawala@gmail.com
}

\begin{abstract}
Introduction
The advent of this pandemic has caused researchers all over the world to study various aspects, from the virus itself, to factors that are affected by it. Psychological research in the wake of COVID19 is no such exception. While mental health concerns during this pandemic are sources of further study for future considerations, we must take into account ethical issues. Privacy and security concerns are of utmost importance, as research in this field often involves sensitive data such as a patient's medical records and psychiatric history. In case of in person research, the researcher should follow all COVID-19 related protocols like masking, social distancing etc. The researcher should also ensure the research participants are following protocol as well. Sensitivity during this pandemic with respect to data is also an important issue. Individuals are going through highly stressful periods, experiencing a downward spiral of mental health, grief, and financial stress. A lot of families have not only had to deal with the loss of their loved ones but have been deprived from saying their last goodbyes and carrying out their funeral rites. Researchers must keep in mind this vulnerable mental state. Health care systems around the world have been crumbling with work pressure and hence it is very important for researchers to be sensitive while conducting research and not press health workers to give out confidential details. Ethical committees must set clear cut guidelines with respect to psychological research, to ensure that the rights of the participants are given utmost importance.
\end{abstract}

\section{Sensitivity during data collection}

Research during the pandemic brings about the concern about sensitivity towards participants. For purposes of research, the researcher's sample could involve grieving families, patients who have been on the ventilator, and vulnerable populations such as those who have serious mental illnesses. It is especially important that the researcher keeps in mind that the participants' mental state has to be protected and taken care of. For example, wording of questions in surveys and interviews should be such that the questions themselves don't trigger a downward spiral of emotions, or make symptoms of their mental illness worse. The researcher must ensure that participants are not reminded of the stressful period that they went through, as it can bring about memories of the traumatic experience. It is imperative that researchers draw the line between the quest for data collection and the very sensitivity that is required on the part of humanity during these times.

Experienced researchers must train their assistants in how to collect data from the population, when the topic of study is sensitive. An atmosphere of trust and emotional support should be built above all, rather than the mechanical collection of data.

In the Indian context, the Indian Council of Medical Research (ICMR) has released ethical guidelines for research during COVID-19. It highlights the possibility of stigmatizing patients who have tested positive, as well as disadvantaged communities. Additional guidelines have been mentioned for those individuals who have gone through particularly stressful experiences. There have been specific guidelines for research on psychology and mental health, and they involve aspects such as the provision of psychosocial support, empathy, and utmost respect. 


\section{Privacy and data storage concerns}

Privacy and security concerns are one of the main ethical issues to be accounted for when undertaking any form of research. Online research raises a lot of concerns of privacy and safety of data. When researchers use online platforms, they must ensure the apps are secure and end to end encrypted and aren't stealing data for marketing, selling data etc. Data collection methods done via video conference platforms such as Zoom, Google Meet, or WhatsApp video calls must ensure that the recordings are stored with utmost safety. Other platforms such as Google Forms must ensure that data is not shared by multiple researchers, to avoid data leakage. The number of people who have access to the data should be kept minimal as greater the number of people who have access to the data, higher the risk of data leaking. When participants take part in a research study, they are trusting the researcher with their information.

In today's world data is extremely valuable and data theft is widespread. Sensitive data, related to both physical and mental health must be protected. In the age of digital leaks and hacking, such data needs to be especially protected. The next issue related to privacy involves the storage of data. Hackers in the past, have been able to get access to patients' medical records at hospitals in a bid to extort money from the hospital. Especially keeping in mind, the context of COVID-19, researchers who have access to patients' medical records must ensure that data is saved with utmost safety and security, using encryption. Researchers need to be careful and make sure their data is stored securely and is hack-proof. A lot of COVID-19 patients have been facing a lot of stigmatization and discrimination. When research studies are conducted, participants often disclose very personal details and hence it is crucial these details stay confidential and the participants are protected from any kind of discrimination or stigmatization. It is the researcher's responsibility to maintain confidentiality and safeguard the participants' information.

Details about a patient's mental health disorders, ranging from anxiety, to schizophrenia must be saved with utmost privacy, after the participant, or the caregiver has provided consent about their data being used for a research study.

To ensure that privacy and confidentiality is maintained at all times, researchers must set clear guidelines on data collection and storage procedures before anything else. Especially if all the data collection is done remotely online, extra security measures should be added wherever possible.

While researchers might be motivated to publish the most recent findings related to the impact of the pandemic on mental health, caution must be taken in ensuring that privacy is not breached. Anonymity must be provided wherever possible.

\section{Safety of researchers and participants in case of in person research methods}

Mental health research is predominantly through face-to-face interview methods, or through observational methods. However, these methods generally involve the researcher and participant being in an outdoor setting which could be potentially dangerous to both as they are risking exposing themselves to the virus. As far as possible, researchers should try to switch to virtual platforms as an alternative medium for conducting research as that greatly mitigates their risks. Since participants could get wary about giving personal data for research through an online medium, researchers could reassure them by addressing privacy concerns by providing them with evidence that the platform they are using is safe and end-to-end encrypted.

In cases where virtual research methods aren't possible, researchers must strictly follow all COVID-19 related protocols like wearing a mask, face shield, maintaining social distance etc. As far as possible, while going to conduct research, the researchers should avoid public transport and try to travel in a private vehicle so that they cut down their exposure. If a group research is involved, it is very important to schedule it in a way that prevents overcrowding and stick to the schedule. While conducting any form of interactive research, the researchers must also ensure the research participants are also following all safety protocols. If participants are found not following safety protocol, for example, lowering their face mask, it is important for the researcher to correct them and immediately request them to follow the safety protocol. 


\section{Research with Vulnerable Populations}

\section{Children and adolescents}

The biggest ethical issue while doing research with children and adolescents is that of consent. Especially with young children or those who are differently abled, it is imperative that the consent of these participants is taken before anything else. UNICEF has released guidelines on ethical considerations while conducting research on violence against children during the COVID-19 pandemic, where they emphasise that the requirement for gathering data should not override the consideration of the risks involved. These ethical guidelines include the possibility of gathering data remotely to eliminate face to face contact, lockdown rules and restriction measures to name a few. Further, many children have lost their parents due to COVID, and are in a particularly vulnerable mental state. Care needs to be taken that such groups are handled with utmost care and sensitivity, with experienced researchers in the field of paediatric research. In many cases, especially with infants and toddlers, the consent of caregivers is mandatory. Education settings like pre-schools, non-governmental organisations, orphanages, and child development clinics will have ethical guidelines of their own which researchers have to keep in mind.

\section{Sensitivity and safety with the geriatric population}

Majority of the geriatric population are not technologically savvy and hence while conducting research with the geriatric population, researchers may be forced to conduct in person research. However, the older population is a high-risk population for Covid-19 as they have a range of comorbidities and weaker immunity than younger people. It is crucial for the researcher to follow safety protocol especially while interacting with the older population.

One of the many ethical issues that come up while conducting research with the home-bound geriatric population is the researcher's role conflict. Parsons has provided a structural account of roles in his notion of the sick role. Merton has also provided information about how role conflict is a sociological construct, in his construct of role-sets. Researcher's role conflict occurs when the researcher is performing two roles with different and sometimes opposing sets of expectations. As a researcher, the normative role expectation is to be someone who is supposed to remain objective and not biased. However, while conducting research, if the researcher comes across something that could be potentially harmful to the participant, the researcher would feel an obligation to act in response to the perceived threat, abuse or neglect of the study participant while at the same time, the researcher would feel the need to remain objective and not do anything that would interfere with the study and dilute the validity of the results.

There are also many ethical issues while conducting research with the geriatric population that suffers from disorders like Alzheimer's. If researchers are conducting research on patients who have recovered from COVID-19, and any of the participants are suffering from Alzheimer's they may have no recollection of it and as a result it may scare them and heighten their paranoia. Many old age related psychological problems like Alzheimer's diminish a person's understanding and decision making abilities. Hence, it is unethical to conduct research on them as they may not fully understand the consent process and their willingness to participate may hence not be entirely consensual.

\section{Ethical issues while doing research on healthcare workers}

Health care systems around the world have been crumbling with pressure due to the COVID-19 pandemic. Health care professionals are being faced with challenges they have never seen before. Besides working themselves to the bone with extra shifts and increased patient load, they also have to worry about their own exposure to the virus and carrying the risk back home to their families. Health care workers also feel a lot of helplessness like in situations where they have to turn patients away, or when a particular treatment isn't working and they are losing the patient. A crosssectional study on the impact of mental health among medical and nursing staff in Wuhan during the 2019 COVID-19 outbreak confirmed two-thirds of mental health disorders, especially in young women. Health care workers form a major cohort for COVID-19 related research, however, there are certain ethics researchers must keep in mind. Researchers should respect confidential data like 
a patient's personal details and not press doctors, nurses etc. for giving out this type of sensitive data while conducting a research.

Around the world there have been shortages of medical resources and hospitals have had to turn away patients as they couldn't cope with the numbers. Globally, a lot of medical staff have reported feeling a moral dilemma while providing care for some patients where they know there is minimal medical benefit for the patient as they are nearing the end. In these cases, they feel particularly conflicted in turning away healthier patients who come in for treatment and have a much stronger chance to survive. All of these added pressures are definitely causing an impact on the mental health of healthcare workers and hence it is extremely important for researchers to acknowledge that these are different times and while conducting research on health care workers they must be very sensitive and respectful.

\section{Suicide}

Suicide in general, is a mental illness where research data is particularly sensitive. Whether it is related to the family of the individual who committed suicide, or the individual themselves who attempted to end their life, sensitivity and an atmosphere of social support is extremely essential. India witnessed a number of suicides related to COVID-19, and Japan saw its rates of suicide increase in 2020, for the first time in over a decade. Research on the topic of suicide is an important aspect of psychological research during COVID-19, as its findings could be particularly important in providing social support and mental health services to suicidal individuals. That being said, the ethical considerations must not be ignored. It is researcher must be extremely sensitive while gathering data, especially in the case of a suicide attempt. Any wrong wording can cause the individual to be reminded of the traumatic experience, and might make matters worse. It is imperative that the victim and the victim's family is given utmost respect and anonymity.

\section{Studies on grief and death ethics}

COVID-19 has taken the lives of millions and left families devastated. A study about the ethical issues in the study of bereavement examined the theoretical opinions of bereaved adults about ethical issues. Participants felt positively about bereavement research, although opinions about timing and method of recruitment were varied. The results also suggest that bereaved individuals should be considered competent to consent to bereavement research participation.

Not only have families struggled with the loss of loved ones, they haven't got closure as many families were not even allowed to meet their deceased relatives and carry out their funeral rites. A documentary study was done to study the effect of suppressing funeral rituals during the COVID19 pandemic on bereaved families. Based on the analysis of the reports collected, they found that in addition to serious harm to the mental and physical health of those affected, Covid-19 also imposes challenges that need to be considered when planning psychosocial interventions. Families suffer due to being impeded from accompanying their loved ones during their final days in the hospital. After a lonely and helpless death, the suspension of funeral rites makes the mourning of the family members even more painful and incomplete. Hence with families dealing with loss and without getting to say their final goodbye, it is crucial for researchers to be extremely sensitive while conducting any kind of study and maintain grief and death ethics. They must also maintain dignity and avoid asking the family to repeat painful details about their loved one's death.

\section{Ethics committees and their role during COVID-19}

Psychological associations all around the world have ethical committees for research. These committees have to create a set of guidelines to protect the rights of the participants, and ensure that the research studies undertaken do not violate any ethical norms set by the committees. In addition, healthcare facilities such as hospitals also have their own ethics committees. These committees might consist of doctors themselves, who come up with a set of clear guidelines. However, in the wake of COVID-19, these doctors are busy on duty, and their focus is entirely shifted to patient care during this time. At a time when researchers want to understand the mental health impacts of the pandemic, these doctors and members of ethical committees might not give the attention required. Therefore, unknowingly or knowingly, researchers might bring in ulterior 
motives and violate the ethical guidelines of the facility, potentially putting their participants at risk.

The WHO has released guidelines for research ethics committees for rapid review of research during public health emergencies in order to ensure that research as well as ethical standards are not compromised. The report states that such guidelines must come into place once a public health emergency is declared, and brings up an important point of conducting virtual meetings to review research processes in the event that face-to-face meetings are not possible. The report also mentions that individuals who are experts in the field of ethics must be contacted in advance to ensure that there is no shortage of committee members. All-in-all, researchers must ensure that they take all the required approvals from the ethical committees of the healthcare facilities whose patients they are using as a part of their sample. Be it mental hospitals, old age homes or psychiatric units, the researchers must ensure that they are not violating any ethical norms of that facility.

\section{RECOMMENDED REFERENCES}

1. Indian Council of Medical Research. National Guidelines for Ethics Committees Reviewing Biomedical and Health Research During COVID-19 Pandemic. 2020. https://ethics.ncdirindia.org//asset/pdf/EC_Guidance_COVID19.pdf

2. Collier K. (2021, February 5). Hackers post detailed patient medical records from two hospitals to the dark web. NBC News. https://www.nbcnews.com/tech/security/hackers-post-detailedpatient-medical-records-two-hospitals-dark-web-n1256887

3. Choudhury S, Ghosh A. Ethical Considerations of Mental Health Research Amidst COVID-19 Pandemic: Mitigating the Challenges. Indian J Psychol Med 2020;42(4):379-81.

4. Research on violence against children during the COVID-19 pandemic: Guidance to inform ethical data collection and evidence generation. UNICEF DATA; 2020

5. Locher JL, Bronstein J, Robinson CO, Williams C, Ritchie CS. Ethical Issues Involving Research Conducted With Homebound Older Adults. The Gerontologist 2006;46(2):160-4.

6. Kang L, Ma S, Chen M, Yang J, Wang Y, Li R, Yao L, Bai H, Cai Z, Xiang Yang B, Hu S., Zhang $\mathrm{K}$, Wang G, Ma C, Liu Z. Impact on mental health and perceptions of psychological care among medical and nursing staff in Wuhan during the 2019 novel coronavirus disease outbreak: A crosssectional study. Brain Behav Immun 2020;87:11-7.

7. Wingfield-Hayes BR. (2021, February 18). Covid and suicide: Japan's rise a warning to the world? BBC News. https://www.bbc.com/news/world-asia-55837160

8. Beck AM, Konnert CA. Ethical Issues in the Study of Bereavement: the Opinions of Bereaved Adults. Death Stud 2007;31(9):783-99.

9. World Health Organization. (2020). Guidance for research ethics committees for rapid review of research during public health emergencies. https://apps.who.int/iris/bitstream/handle/10665/332206/9789240006218-eng.pdf?ua=1

Acknowledgements: Nil

Funding: Nil

Conflict of interest: Nil 